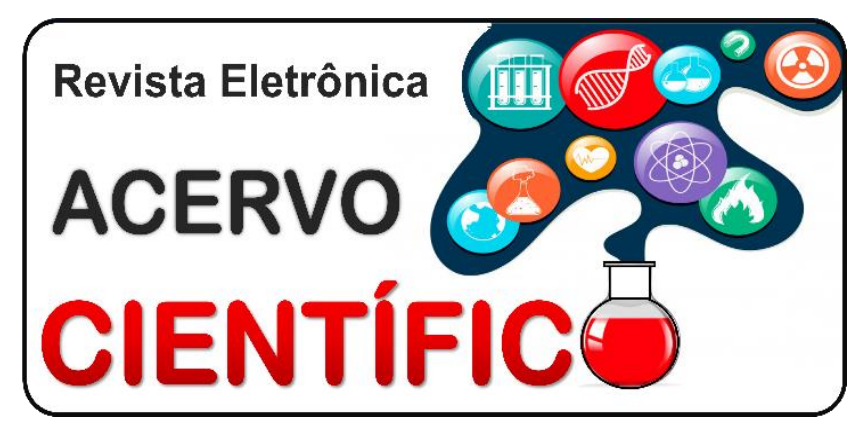

REVISÃO BIBLIOGRÁFICA

Recebido em: 11/2020

Aceito em: 12/2020

Publicado em: 2/2021

\title{
Comparação das Técnicas Lichtenstein e Reparo Assistido por Robô para Herniorrafia Inguinal: uma revisão bibliográfica
}

\author{
Comparison of the Lichtenstein and Robot Assisted Repair Techniques for Inguinal \\ Herniorraphy: a literature review
}
Comparación de las técnicas de Lichtenstein y la reparación asistida por robot para la herniorrafia inguinal: revisión de la literatura

Tainara Sales Miranda ${ }^{1 *}$, Aline Trovão Queiroz ${ }^{2}$, Andressa Neves Rebello Dyna ${ }^{3}$, Camila Ferreira Mariani $^{4}$, Deocárdio Cardoso Souto da Conceição ${ }^{5}$, Elisiane Rodrigues Garioli ${ }^{4}$, Leonardo Paiva Martins $^{4}$, Letícia Cuzzuol Lyra ${ }^{4}$, Rafael dos Santos Borges ${ }^{6}$, Rafael Veloso Starling Schwanz ${ }^{4}$.

\begin{abstract}
Resumo: O presente estudo visa comparar as técnicas de Lichtenstein, padrão-ouro das técnicas abertas, de cirurgia de herniorrafia inguinal, realizada de forma livre de tensão e utilizando tela, e a técnica de Reparo Assistido por Robô (R-IHR), que se utiliza dos princípios cirúrgicos da técnica laparoscópica minimamente invasiva, permitindo reparo totalmente extraperitoneal, ou peritoneal transabdominal. As técnicas foram comparadas em relação complicações no geral, dor relacionada aos procedimentos, seromas e hematomas, infecção, inguinodinia, recidiva, curva de aprendizado, tempo de recuperação, reprodutibilidade e custo-benefício. Assim, observou-se que a técnica R-IHR apresenta vantagens como menor número de complicações e menos taxa de infecções, contudo, a mesma mostrou maior custo e maior incidência de inguinodinia, além de maior complexidade de aprendizado. Logo, corroborando com a premissa da Sociedade Brasileira de Hérnia, não existe uma técnica de herniorrafia universal. Devendo-se avaliar o método quanto às variáveis apresentadas e seu custo-benefício para a melhor escolha da técnica.
\end{abstract}

Palavras-chave: Herniorrafia, Procedimentos Cirúrgicos Robóticos, Hérnia Inguinal.

\footnotetext{
Abstract: The present study aims to compare the Lichtenstein technique, the gold standard of open techniques of inguinal herniorrhaphy surgery, performed in a tension-free manner using a screen, and the Robot Assisted Repair technique (R-IHR), which uses the surgical principles of the minimally invasive laparoscopic technique, allowing totally extraperitoneal, or transabdominal peritoneal repair. The techniques were compared regarding complications in general, pain related to procedures, seromas and bruises, infection, inguinodynia, recurrence, learning curve, recovery time, reproducibility and cost-benefit. Thus, it

${ }^{1}$ Centro Universitário de Caratinga (UNEC), Caratinga-MG. *E-mail: tainarasmiranda@hotmail.com

2Universidade de Vassouras (UV), Vassouras-RJ.

${ }^{3}$ Centro Universitário Redentor (UNIREDENTOR), Itaperuna-RJ.

${ }^{4}$ Universidade Vila Velha (UVV), Vila Velha-ES.

5 Universidade Cidade de São Paulo (UNICID), São Paulo-SP.

'Universidade Federal de Minas Gerais (UFMG), Belo Horizonte-MG.
} 
was observed that the R-IHR technique has advantages such as fewer complications and less infection rate, however, it showed a higher cost and a higher incidence of inguinodynia, in addition to greater learning complexity. Therefore, corroborating the premise of the Brazilian Hernia Society, there is no universal herniorrhaphy technique. The method and variables should be evaluated and its cost-benefit for the best choice of technique.

Keywords: Herniorrhaphy, Robotic Surgical Procedures, Inguinal Hernia.

Resumen: El presente estudio tiene como objetivo comparar las técnicas de Lichtenstein, el gold standart de las técnicas abiertas de cirugía de herniorrafia inguinal, hecha sin tensión, utilizando una malla, y la técnica de Reparación Asistida por Robot (R-IHR), que utiliza los principios quirúrgicos de la técnica laparoscópica mínimamente invasiva, y permite una reparación totalmente extraperitoneal o peritoneal transabdominal. Las técnicas fueron comparadas en relación a las complicaciones en general, dolor relacionado con los procedimientos, seromas y hematomas, infección, inguinodinia, recurrencia, curva de aprendizaje, tiempo de recuperación, reproducibilidad y costo-beneficio. Así, se observó que la técnica RIHR tiene ventajas como menos complicaciones y menor tasa de infección. Sin embargo, la misma mostró un mayor costo y una mayor incidencia de inguinodinia, además de una mayor complejidad de aprendizaje. De tal manera, corroborando com la premisa de la Sociedad Brasileña de Hernia, no existe una técnica de herniorrafia universal. El método debe ser evaluado por suyas variables y su costo-beneficio para la mejor elección de técnica.

Palabras clave: Herniorrafia, Procedimientos Quirúrgicos Robotizados, Hernia Inguinal.

\section{INTRODUÇÃO}

A correção de hérnia inguinal é um dos procedimentos médicos cirúrgicos mais realizados no mundo, ultrapassando 20 milhões de operações. No Brasil, de março de 2015 a março de 2016, o Sistema Único de Saúde realizou 117.090 hérnias inguinais unilaterais e 901 casos foram realizados por via laparoscópica (IRANIHA A e PELOQUIN J 2017; PEDROSO LM, et al., 2017).

Desde 1887, mais de 70 métodos de reparo de hérnias inguinais foram introduzidos e, desde o advento da cirurgia laparoscópica, em meados da década de 1990, os métodos de reparo de hérnias inguinais foram desenvolvidos gradualmente (IRANIHA A e PELOQUIN J, 2017). Contudo, apesar da introdução da correção de hérnia laparoscópica, a correção aberta ainda é amplamente utilizada (KOSTURAKIS AK, et al., 2018).

Atualmente, apenas três tecnologias foram comprovadas cientificamente e podem ser recomendadas para uso clínico. Com o advento da cirurgia assistida por robô, aumentou-se o interesse na viabilidade e nos resultados relacionados à aplicação da tecnologia robótica na cirurgia geral, incluindo o reparo de hérnias inguinais. O reparo robótico de hérnia inguinal $(\mathrm{R}-\mathrm{IHR})$ oferece as vantagens de um método minimamente invasivo, e a robótica oferece vantagens sobre a laparoscopia (KOSTURAKIS AK, et al., 2018).

O aumento da demanda por estética pós-operatória tem estimulado o interesse das pessoas pela cirurgia de portal único (cirurgia de ponto único laparoscópica, LESS). A plataforma do robô agrega ao laparoscópio à riqueza de movimento, a conveniência de operação e procedimentos, a visão tridimensional e a ergonomia do cirurgião, agregando assim, as vantagens da cirurgia robótica ao procedimento LESS (BOSI HR, et al., 2016).

Embora o reparo laparoscópico de hérnias inguinais (L-IHR) tenha vantagens conhecidas, incluindo redução da dor pós-operatória precoce, redução da permanência hospitalar e redução de infecções de feridas, poucas publicações estudaram os benefícios potenciais de R-IHR (BITTNER IV, et al., 2016).

Os supostos benefícios da cirurgia robótica de hérnia inguinal incluem redução da dor pós-operatória associada à rede de sutura usada para fixação (em oposição à fixação de pinos em reparos laparoscópicos 
tradicionais) e ergonomia melhorada (posicionamento corporal e posicionamento durante a cirurgia) (PRABHU AS, et al., 2020).

Diante do exposto, o objetivo do presente estudo consiste, por meio de uma revisão narrativa, comparar a técnica de reparo de hérnia inguinal aberta com a técnica de Lichtenstein, com o promissor reparo assistido por robô (R-IHR).

\section{REVISÃO BIBLIOGRÁFICA}

\section{Hérnias inguinais}

Hérnia é definida como protrusão anormal de um tecido ou órgão por um defeito em suas paredes adjacentes. Podem acontecer em diversos locais do corpo, mas esse defeito é mais frequentemente relacionado com a parede abdominal, sendo a região inguinal a mais acometida (75\% de todas hérnias) (TEIXEIRA FMC, et al., 2017).

As hérnias inguinais podem ser diretas ou indiretas, com uma incidência de dois terços serem indiretas e o restante diretas. As indiretas descem ao longo do canal inguinal, tendo um caráter congênito, já as diretas decorrem da protrusão através do triângulo de Hesselbach, decorrente de uma fraqueza na fáscia transversal sendo relacionada a defeitos hereditários na síntese ou renovação do colágeno (TEIXEIRA FMC, et al., 2017).

Figura 1 - llustração representativa de hérnia inguinal.

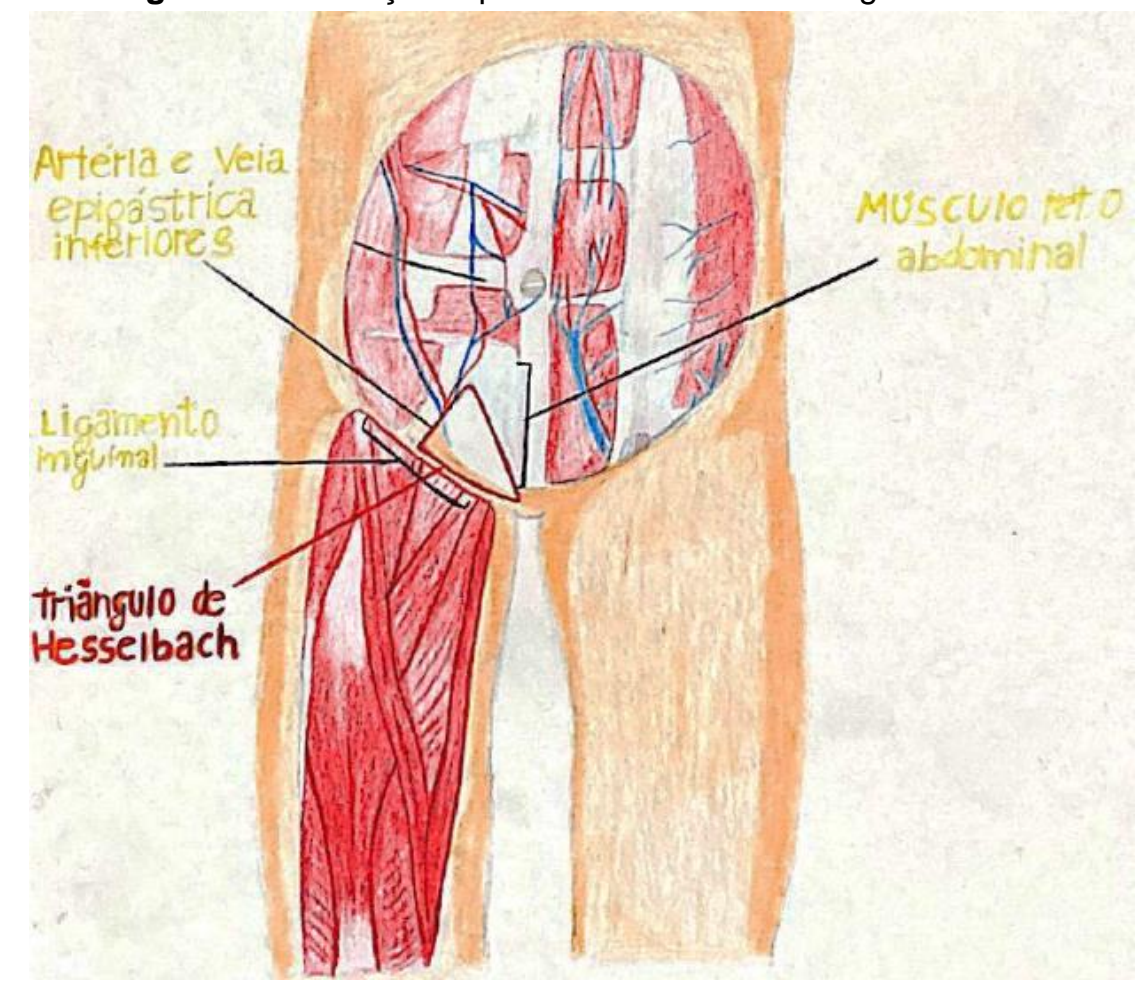

Fonte: Miranda TS, et al., 2020. Adaptada de: Hérnia Inguinal: Anatomia, Patofisiologia, Diagnóstico e Tratamento Goulart A e Martins S, 2015. Ilustração cedida por Mariana Camilly Campos Miranda, 2021.

As hérnias inguinais são mais comuns em crianças (SHI Y, et al., 2019), porém por sua alta incidência faz-se importante também no adulto. Há uma relação de um a cada quatro homens americanos adultos que correm risco de desenvolver hérnia inguinal (HUERTA S, et al., 2019). Em comparação aos gêneros, a incidência cumulativa é de $27 \%$ em homens e $3 \%$ em mulheres. Além disso, o risco aumenta com a idade. (HUERTA S, et al., 2019). 
O tratamento de escolha primária para hérnia inguinal é o reparo cirúrgico (SHI Y, et al., 2019), e aproximadamente 20 milhões de hernioplastias inguinais são realizadas em todo o mundo anualmente, levando a um custo significativo e a uma grande carga de morbidade ao redor do mundo (SHI Y, et al., 2019). Assim, todos os aspectos que poderiam minimizar complicações e custos devem ser bem analisados. Portanto, as técnicas que serão comparadas são Lichtenstein (cirurgia aberta) e o reparo assistido por robô (R-IHR).

\section{Lichtenstein}

A técnica de Lichtenstein consiste em um reparo livre de tensão, utilizando-se tela e é consagrada como padrão ouro das técnicas abertas, dados seus resultados clínicos excelentes e simplicidade no aprendizado e reprodução (CLAUS et al, 2019), além de demonstrar oferecer menos dor pós-operatória e possibilidade de reintegração mais rápida ao trabalho ao se comparar com as técnicas sem tela (PALERMO $\mathrm{M}$, et al., 2015).

No procedimento, após a incisão e abertura da parede abdominal por planos, realiza-se a dissecção do canal inguinal, seguida de ligadura alta de saco herniário indireto, rebatendo-se inferiormente as estruturas do cordão espermático. Progride-se, então, para o isolamento e tração cranial da aponeurose do músculo oblíquo externo, de maneira que o músculo oblíquo interno subjacente, suporte uma tela de 6 a 8 centímetros de extensão, sobrepondo-se, necessariamente, a borda do músculo oblíquo interno por 2 a 3 centímetros (SABISTON DCJ, 2014).

Coloca-se, então, sobre o canal inguinal, a tela, que deve ser plana de polímero monofilamentar, como o polipropileno, com gramatura entre 30 e $140 \mathrm{~g} / \mathrm{m} 2$ e com poro maior do que $1 \mathrm{~mm}$, cujas características são recomendadas pela Sociedade Brasileira de Hérnia por impactarem em melhores desfechos no que concerne à dor, sensação de corpo estranho, resistência à infecção e formação de tecidos fibróticos com consequente diminuição da superfície de tela (CLAUS CMP, et al., 2019). Deve-se atentar, ao colocar-se a tela, para seccionar a margem distal e lateral da tela de forma a abrigar o cordão espermático, rebatendo-o cefalicamente, e ainda, para garantir uma margem de tela de cerca de 2 centímetros que ultrapasse o osso púbico (SABISTON DCJ, 2014).

Segue-se para a fixação, com fio monofilamentar inabsorvível, da tela no tecido aponeurótico, que reveste medialmente o osso púbico, suturando-o ao tendão conjunto. Inferolateralmente, fixa-se a malha ao ligamento inguinal continuamente até atingir as adjacências do anel inguinal interno (SABISTON DCJ, 2014). As pontas construídas pela abertura previamente realizada na tela são suturadas uma à outra, confeccionando um novo anel inguinal interno, acomodando o cordão espermático. Sintetiza-se a aponeurose do músculo oblíquo externo sobre o cordão espermático e realiza-se as demais etapas de fechamento por planos (SABISTON DCJ, 2014).

\section{Reparo assistido por robô (R-IHR)}

O reparo robótico de hérnia inguinal utiliza os mesmos princípios cirúrgicos da técnica laparoscópica minimamente invasiva. Tanto a abordagem robótica totalmente extraperitoneal (rTEP) como o reparo peritoneal transabdominal robótico (rTAPP) são possíveis, sendo o rTAPP comumente utilizado e, por isso, será o método descrito nesta sessão (PODOLSKY D e NOVITSKY Y, 2020).

O rTAPP é útil em pacientes com história de prostatectomia aberta, procedimentos pélvicos, procedimentos interno abdominais, além de pacientes obesos. A única contra-indicação absoluta é a intolerância à anestesia geral ou pneumoperitônio. Entretanto, alguns outros fatores podem aumentar o risco de complicações pós-operatórias; como abdômem hostil, cirurgia pélvica extensiva anterior e ostomia. Digno de nota que a presença de uma tela pré-peritoneal prévia aumenta a dificuldade da cirurgia e preferencialmente deve ser abordada por um cirurgião experiente (PODOLSKY D e NOVITSKY Y, 2020).

Para realização do procedimento, o paciente é posicionado em decúbito dorsal e em Trendelenburg. Em seguida, é feita antibioticoprofilaxia com cefalosporina de primeira geração, como a Cefazolina. Utiliza-se uma perneira no paciente e o robô é acoplado (BOSI HR, et al., 2016). 
Realiza-se uma incisão umbilical para inserção do trocarte específico para portal único robótico, que tem por característica a entrada cruzada das pinças (BOSI HR, et al., 2016). Prossegue-se com incisão curvilínea no peritônio, acima do defeito herniário, entre o ligamento umbilical mediano e a espinha ilíaca anterosuperior (PIROLLA EH, et al., 2018).

O próximo passo é a dissecção peritoneal, a qual precisa ser muito cuidadosa para não lesar os vasos epigástricos inferiores (PIROLLA EH, et al., 2018). Prossegue-se para a redução do saco herniário. Para herniações indiretas, nas quais o saco localiza-se anterolateralmente, utiliza-se dissecção romba e eletrocautério para separá-lo das estruturas testiculares. Nos defeitos diretos, utiliza-se simplesmente dissecção romba para apreender e reduzir o saco herniário (PODOLSKY D e NOVITSKY Y, 2020).

Em seguida, é feita a colocação da tela. O uso de um trocarte de $12 \mathrm{~mm}$ é indicado por facilitar a colocação da tela escolhida e possibilitar o uso de grampeadores com diâmetros maiores e clipes de fixação especiais para pacientes super obesos. Em relação aos tipos de telas utilizadas, os mais citados são a tela auto fixável (Progrip $®$ ), a tela de polipropileno e a tela de poliéster auto fixável; sendo recomendada sua fixação com sutura absorvível ou grampos absorvíveis (PIROLLA EH, et al., 2018). Ainda, é preciso evitar qualquer fixação nas áreas ricas em estruturas neurovasculares (PODOLSKY D e NOVITSKY Y, 2020).

Finalmente, o espaço pré-peritoneal é fechado com grampos. Terminado o procedimento o robô é desacoplado e o portal umbilical é retirado (PIROLLA EH, et al., 2018). As principais complicações pósoperatórias incluem retenção urinária, seroma e hematomas, com taxa geral de complicações de 7,5\%; sendo a recorrência dos casos semelhantes à via laparoscópica convencional (AIOLFI A, et al., 2019).

\section{Variáveis comparativas específicas entre as técnicas}

Segundo a Sociedade Brasileira de Hérnia, não existe uma técnica de herniorrafia universal. Portanto, ao escolher, deve-se avaliar o método quanto aos índices de complicação, curva de aprendizado, tempo de recuperação, reprodutibilidade dos resultados e o seu custo-benefício (CLAUS CMP, et al., 2019). A incidência de complicações do reparo robótico é de 2,7\% em comparação com a taxa de $11,5 \%$ detectada em reparos tradicionais (PIROLLA EH, et al., 2018).

Em um estudo que analisou a percepção de dor dos pacientes, o grupo que realizou cirurgias robóticas, obteve de maneira significativa uma redução na dor aguda no pós-operatório e dos dias de analgésicos prescritos, quando comparada com as cirurgias abertas. Além disto, pacientes que já haviam realizado uma herniorrafia prévia, relataram uma duração menor da dor na cirurgia robótica (BITTNER IV, et al., 2018).

Segundo Aiolfi A, et al. (2019), seroma e hematoma são algumas das complicações pós-operatórias mais comuns e apresentam taxas de incidência iguais, independentemente da técnica realizada. As taxas de infecção são maiores no pós-operatório das técnicas abertas, enquanto nas cirurgias robóticas, estas tendem a não ocorrer. Quando ocorrem, é devido ao grande tempo cirúrgico (POKALA B, et al., 2019; PIROLLA EH, et al., 2018). No que se diz a retenção urinária, orquite isquêmica e outras complicações importantes tais como pneumonia por aspiração, infarto agudo do miocárdio, trombose venosa e perfuração gastrointestinal a cirurgia robótica apresentou uma maior taxa quando comparada a cirurgia aberta (HUERTA S, et al., 2019).

Segundo Huerta S, et al. (2019), em sua análise retrospectiva, apesar da taxa de inguiniodinias variar amplamente na literatura (1 a 50\%), existe um consenso de uma incidência de 10\%. Tal consenso contrasta com o seu achado de que a ocorrência de inguinodinia é significantemente maior na cirurgia robótica em comparação com a aberta $(1.5 \%$ versus $14.1 \%, p<0.001)$. A taxa de recorrência das abordagens disponíveis é de $2 \%$, sendo que as taxas de recorrência são similares quando comparadas entre si, exceto quando se compara a robótica (5.6\%) com a aberta (1.7\%). Já em estudos com um follow-up curto, de 16 a 365 dias, a taxa de recorrência seria de $0,18 \%$ (AIOLFI A, et al., 2019).

Segundo Pokala B, et al. (2019), após a retirada de um estudo em uma metanálise, a taxa de recorrência e de dor crônica não foi diferente entre a Técnica Lichtenstein e a robótica. Digno de nota que mesmo possuindo uma frequência menor que as demais a dor inguinal crônica é uma das complicações principais, 
mas que possui alivio sintomático com compressas quentes, compressão externa e pelo uso de drogas antiinflamatórias não esteroidais (PODOLSKY D e NOVITSKY Y, 2020). No que se diz quanto a curva de aprendizado da técnica robótica esta apresenta um ponto de inflexão de aproximadamente 12 casos, enquanto a técnica de Lichtenstein é fácil de ser replicada e ensinada em todos os níveis (JANJUA H, et al., 2020; CAMPANELLI G, et al., 2017).

A facilidade da curva de aprendizado na Técnica de Linchtenstein é percebida facilmente quando 4 a 5 casos, por cirurgião, em um mutirão, consegue gerar uma mudança significativa nos resultados quanto à incisão, dissecção, preparação, corte da tela, fixação da tela, manejo dos instrumentos, respeito pelos tecidos, tempo e flow cirúrgico, fechamento e classificação geral (LAZZARINI-MENDES C, et al., 2016). Digno de nota que a curva de aprendizado na transição aberta e robótica é maior do que quando comparado à transição laparoscópica - robótica (POKALA B, et al., 2019). Para efeito de comparação, a curva de aprendizado da técnica endoscópica pode chegar a até 50 a 100 casos, sendo os primeiros 30 a 50 casos os mais críticos (CAMPANELLI G, et al., 2017).

Segundo Almarzooqi R, et al. (2019), apesar de se ter a convicção de que as técnicas minimamente invasivas possuem o tempo de recuperação mais rápido quando realizadas por cirurgiões experientes na presença dos recursos necessários, se concluiu nos guidelines da Sociedade Europeia de Hérnia, que quando comparadas com as técnicas abertas de cirurgia, os resultados foram comparáveis em um follow-up de 2 anos. Apesar disto, para Bittner IV, et al. (2018); os pacientes percebem uma menor interrupção de suas atividades físicas que requerem esforço físico na primeira semana.

O tempo médio de retorno de recuperação para reinserção no trabalho é de cerca de 30 dias para hernioplastias com tela, contra 90 dias para aquelas sem tela (PALERMO M, et al., 2015). De acordo com Iraniha A e Peloquin J (2017), Tapp assistido de cirurgia robótica, possui uma boa reprodutibilidade devido à sua curva de aprendizado e segurança. Ao se analisar o custo-benefício de uma técnica, é necessário ter em mente, que o custo total inclui o custo fixo, composto pelos gastos com os funcionários, e o custo capital, por exemplo o aparelho robótico em si, e o custo variável, também chamado de custo direto, composto pelos materiais descartáveis e o equipamento reutilizável (JANJUA H, et al., 2020; WAITE K, et al., 2016).

Dentre os estudos analisados, é digno de nota que não houve variação significante entre os preços cobrados pelos cirurgiões, porém os custos totais e fixo hospitalar da cirurgia robótica, respectivamente apresentam aumento significativo $(p<0.001)$ quando comparados com às técnicas laparoscópica (CHARLES $\mathrm{E}$, et al., 2017). Em um estudo que analisou apenas o custo direto a técnica robótica foi a mais cara ( $p \leq 0.001$ ), seguida das técnicas de via aberta OHIR e a mais barata de todas era a LIHR (POKALA $B$, et al., 2019). Segundo Aiolfi A, et al. (2019); o tempo operatório médio para o reparo unilateral e bilateral utilizando o robô é de 99.5 minutos, mas varia entre 70 e 120 minutos conforme o estudo devido a divergência da definição de tempo operatório. Ao se comparar o tempo operatório com a técnica aberta, percebe-se que cirurgia laparoscópica e robótica apresentam respectivamente um tempo 19\% e 79,3\% maior. Digno de nota que este achado permanece mesmo após o período da curva de aprendizado das técnicas (HUERTA S, et al., 2019).

\section{CONSIDERAÇÕES FINAIS}

Por meio da análise bibliográfica deste estudo, conclui-se que a técnica de R-IHR apresenta consideráveis vantagens em relação à técnica aberta de Lichtenstein, no que diz respeito à menor incidência de complicações, menor percepção de dor pelos pacientes, uso diminuído de analgésicos e menores taxas de infecção. Algumas variáveis analisadas não apresentaram diferença significativa, foram elas: reprodutibilidade e tempo de recuperação. A variável de comparação "Recorrência" apresentou resultados divergentes nos estudos analisados, com um estudo demonstrando maior incidência na técnica robótica e um estudo não demonstrando diferença entre as técnicas. Por outro lado, a R-IHR demonstrou maior incidência de inguinodinia, maior custo total e maior complexidade da curva de aprendizado, o que não reflete diretamente na eficácia da técnica. 


\section{REFERÊNCIAS}

1. AIOLFI A, et al. Primary inguinal hernia: systematic review and Bayesian network meta-analysis comparing open, laparoscopic transabdominal preperitoneal, totally extraperitoneal, and robotic preperitoneal repair. Hernia, 2019; 23(3): 473-484.

2. ALMARZOOQI R, et al. Review of inguinal hernia repair techniques within the Americas Hernia Society Quality Collaborative. Hernia, 2019; 23(3): 429-438.

3. BOSI HR, et al. Local único assistido por robótico para reparo bilateral de hérnia inguinal. Arquivos Brasileiros de Cirurgia Digestiva, 2016; 29 (2): 109-111.

4. CAMPANELLI G, et al. Primary inguinal hernia: The open repair today pros and cons. Asian Journal of Endoscopic Surgery, 2017; 10(3): 236-243.

5. CHARLES E, et al. Inguinal hernia repair: is there a benefit to using the robot? Surgical Endoscopy, 2017; 32(4): 2131-2136.

6. CLAUS CMP, et al. Orientações da Sociedade Brasileira de Hérnia (SBH) para o manejo das hérnias inguinocrurais em adultos. Rev Col Bras Cir, 2020; 46(4).

7. HUERTA S, et al. Huerta Hernia Repair: Outcomes and Predictors of Complications. Journal Of Surgical Research, 2019; 241:119-127.

8. IRANIHA A e PELOQUIN J. Long-term quality of life and outcomes following robotic assisted TAPP inguinal hernia repair. Journal of Robotic Surgery, 2017; 12(2): 261-269.

9. IV BJG, et al. Correction to: Patient perceptions of acute pain and activity disruption following inguinal hernia repair: a propensity-matched comparison of robotic-assisted, laparoscopic, and open approaches. Journal of Robotic Surgery, 208; 13(1): 191-191.

10. IV BJG, et al. Patient perceptions of acute pain and activity disruption following inguinal hernia repair: a propensitymatched comparison of robotic-assisted, laparoscopic, and open approaches. Journal of Robotic Surgery, 2018; 12(4): 625-632.

11. JANJUA H, et al. Robotic Approach to Outpatient Inguinal Hernia Repair. Journal of the American College of Surgeons, 2020; 231(1): 61-72.

12. KOSTURAKIS AK, et al. First 100 consecutive robotic inguinal hernia repairs at a Veterans Affairs hospital. Journal of Robotic Surgery, 2018; 12(4): 699-704.

13. LAZZARINI-MENDES C, et al. Systematic training model for teaching, development and training of instructors in inguinal hernia treatment using the Lichtenstein technique. Revista do Colégio Brasileiro de Cirurgiões, 2016; 43(5): 382-391.

14. PALERMO M, et al. Hernioplastia com e sem tela: Análise das complicações imediatas em um ensaio clínico randomizado controlado. Arquivo Brasileiro de Cirurgia Digestiva, 2015; 28(3):157-160.

15. PEDROSO LM, et al. Estudo comparativo da dor pós-operatória entre lichtensteína e técnicas cirúrgicas de laparoscopia para o tratamento da hérnia inguinal primária unilateral. Arquivos Brasileiros de Cirurgia Digestiva, 2017; 30(3): 173-176.

16. PIROLLA EH, et al. Inguinal Repair Via Robotic Assisted Technique: Literature Review. Arquivos Brasileiros de Cirurgia Digestiva, 2018; 31(4): 1408.

17. PODOLSKY D e NOVITSKY Y. Robotic Inguinal Hernia Repair. The surgical clinics of North America, 2020; 100(2): 409-415.

18. POKALA B, et al. Minimally invasive inguinal hernia repair is superior to open: a national database review. Hernia, 2019; 23(3): 593-599.

19. PRABHU AS, et al. Robotic Inguinal vs Transabdominal Laparoscopic Inguinal Hernia Repair. JAMA Surgery, 2020.

20. SABISTON DCJ. Tratado de cirurgia: A base Biológica da prática Cirúrgica Moderna. 19a Ed. Rio de Janeiro: Elsevier, 2014.

21. SHI Y, et al. Comparison of the e ect of mesh-plug, Lichtenstein, transabdominal preperitoneal, and totally extraperitoneal hernia repair: A network meta-analysis. Rev Assoc Med Bras, 2020; 66(5): 687-691.

22. TEIXEIRA FMC, et al. Estudo de revisão da cirurgia de hernioplastia inguinal: técnica de Lichtenstein versus laparoscópica. Revista Médica de Minas Gerais, 2017; 27: 1860.

23. WAITE K, et al. Comparison of robotic versus laparoscopic transabdominal preperitoneal (TAPP) inguinal hernia repair. Journal of Robotic Surgery, 2016; 10(3): 239-244.

24. AIOLFI A, et al. Robotic inguinal hernia repair: is technology taking over? Systematic review and meta-analysis. Hernia, 2019; 23(3): 509-519. 